\title{
Rehabilitation Therapy for Better Control of Critically III Patients
}

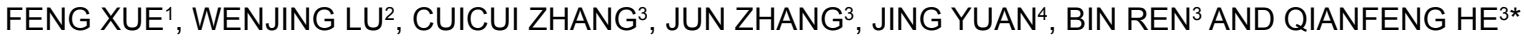 \\ ${ }^{1}$ Medical Department, ${ }^{2}$ Cardiovascular Medicine Department, ${ }^{3}$ Emergency Department, Tangdu Hospital, Air Force Military \\ Medical University, Xi'an, Shaanxi 710038, China ${ }^{4}$ Department of Orthopedics, Armed Police Engineering University Hospital, \\ Xi'an, Shaanxi 710086, China
}

Xue et al.: Rehabilitation treatment for critically ill patients

\begin{abstract}
To explore the value of early rehabilitation therapy in critically ill patients managed on routine treatment, 80 critically ill patients in ICU were randomly divided into the rehabilitation group and the routine treatment group. Each group consisted of $\mathbf{4 0}$ patients. All patients were placed in a recumbent and good limb position. They were turned over and their back was patted every $2 \mathrm{~h}$. According to the patients' condition, they were given symptomatic support treatment such as antiinflammatory agents. Rehabilitation treatment group patients also receive early rehabilitation intervention by a professional rehabilitation team. The results of the ICU hospitalization time and mechanical ventilation time of the two groups, when analyzed, it was found that rehabilitation therapy significantly shortened the ICU hospitalization time and mechanical ventilation time of the patients with significant different. The BI index of the rehabilitation treatment group and the conventional treatment group is significantly increased on the 28 th day after treatment, and the rehabilitation treatment group is better than the conventional treatment group. The APACHE II scores of patients in rehabilitation group and routine treatment group are significantly lower than those before treatment $(\mathrm{P}<\mathbf{0 . 0 5})$. Relevant conclusions were drawn that early rehabilitation by professional rehabilitation team can significantly reduce the duration of mechanical ventilation and ICU hospitalization in critically ill patients. Early rehabilitation treatment did not increase the 28-day mortality rate and the incidence of adverse events, but improved the basic living ability of critically ill patients.
\end{abstract}

Key words: Comprehensive ICU, early rehabilitation therapy, APACHE II score, self-care ability of life

Severe patients often have two or more organ dysfunction or failure, instability of vital signs, which may endanger the lives of patients at any time ${ }^{[1]}$. Since the condition of these patients is often more serious and changes quickly, the current medical staff for critically ill patients in the intensive care unit (ICU) usually use supine position, or even sometimes give restraint band brake, sedative analgesics to reduce tissue oxygen consumption in critically ill patients. However, long-term restriction of physical activities and inadequate passive activities can cause serious harm to various physiological functions especially in critically ill patients ${ }^{[2]}$, such as hypoplastic pneumonia, skin compression or pressure sores at the bone processes. It can also lead to secondary atrophy of skeletal muscle, progressive decrease of limb muscle strength and muscle capacity, resulting in limb dysfunction. It can also cause serious adverse events, such as postural hypotension, arrhythmia, and cardiac dysfunction, which seriously affect the prognosis of patients ${ }^{[3]}$. Rehabilitation originally means recovery and regaining ability. In modern medicine, rehabilitation mainly refers to the recovery of physical and mental function, professional ability and social life ability ${ }^{[4]}$. It aims at reducing or eliminating all kinds of functional disorders caused by diseases, as well as the follow-up effects of functional disorders on the body. According to the actual degree of functional disorders and the needs of patients themselves and the potential of the body, targeted treatment programs are adopted to improve the degree of various functional disorders of patients to the greatest extent, improve the quality of life of patients and improve the daily life and social life of patients. All kinds of skills in action are beneficial for patients to return to their families, jobs and society with few or no obstacles in the early stage ${ }^{[5,6]}$. 
In this study, the method of intervention treatment was adopted to evaluate patient's condition comprehensively, formulate individualized rehabilitation diagnosis and treatment plan to provide progressive comprehensive early rehabilitation intervention measures. The rehabilitation treatment plan is revised in time according to patient's consciousness, tolerance and condition changes. In addition to routine treatment, the value of applying early rehabilitation therapy in comprehensive ICU patients is explored to evaluate the effect of early rehabilitation treatment on infection prevention, control and prognosis of critically ill patients.

\section{MATERIALS AND METHODS}

\section{Selection and exclusion criteria of experimental subjects:}

Participants in the study were adults, aged $18 \mathrm{y}$ and above, who meet APACHE II score $\geq 15$. $24 \mathrm{~h}$ after onset, patients with stable vital signs under the support of drug treatment, instruments and other organs. Patients with first-episode stroke, who were conscious, have no sensory aphasia, cognitive impairment and deafness were included. According to the New York Heart Association, (NYHA), patients' cardiac function is below class IV, as shown in Table 1. Informed consent was signed by either the patient or their family members and this study was approved by the medical ethics committee of Tangdu Hospital, Air Force Military Medical University.

A total of 300 people were selected in this study, 220 cases were excluded according to exclusion criteria. Finally, 80 cases were selected, as shown in fig. 1. The exclusion criteria were, patients with advanced malignant tumors or who have received radiotherapy and chemotherapy for nearly 6 mo, patients with unsound limbs and new fractures without internal fixation, patients with long-term inability to move independently before onset, patients with longterm mechanical ventilation required for neuromuscular lesions.

\section{Research objective:}

According to the above two criteria, 80 critical patients who meet the requirements in comprehensive ICU from July 2014 to July 2018 were included in this study. A convenient sampling method was adopted. According to the formula of sample size, the required sample size was calculated by comparing the sample rate with the total rate. $\alpha=0.05, \beta=0.1$. The formula used was,

$\mathrm{n}=\Pi_{0}\left(1-\Pi_{0}\right)\left[\frac{\mu_{\alpha}+\mu_{\beta}}{\delta}\right]^{2}$

Selected patients were divided into rehabilitation treatment group and conventional treatment group, with 40 cases in each group. There were 21 males and 22 females in the rehabilitation group. The age range was 55-78 y with an average age of 64 . $6 \pm 6$ y. At admission, the body mass index (BMI) was $23.7 \pm 2.1$ and the APACHE II score was $18 \pm 2.5$. In the conventional treatment group, there were 23 males and 20 females, age ranging from 55-80 y, with an average age of $65 \pm 6.6 \mathrm{y}$. At admission, BMI and APACHE II scores were $24 \pm 1.98$ and $18.58 \pm 2.5$, respectively. The general data such as gender, age, BMI, main diagnosis and APACHE II scores of the two groups at admission were analyzed and were found to be not different as shown in Table 2.

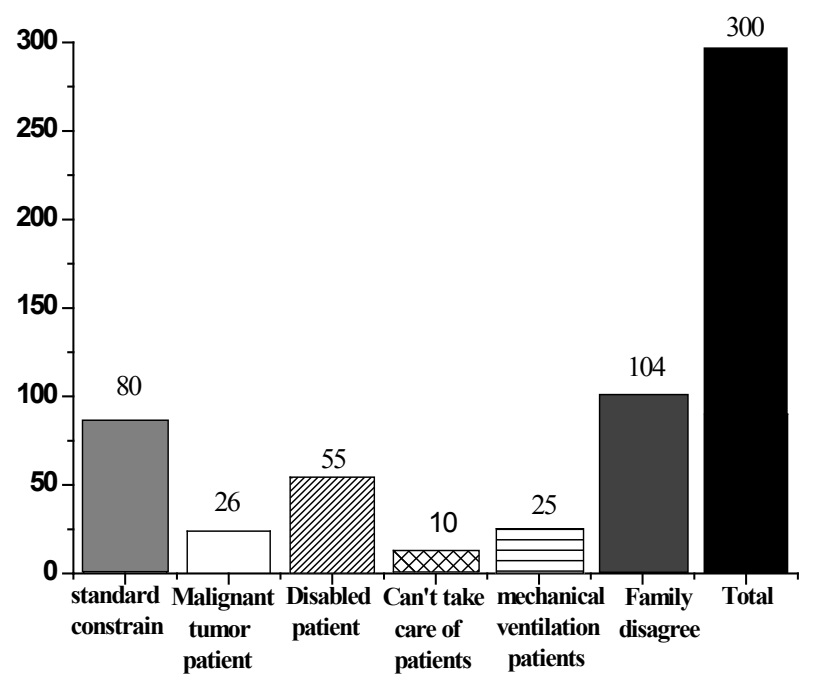

Fig. 1: Experimental primary selection

\section{TABLE 1: NYHA GRADING STANDARDS}

\begin{tabular}{ll}
\hline Grading & Symptom \\
I & $\begin{array}{l}\text { Unrestricted activities, daily physical activity does not cause obvious shortness of breath, fatigue or palpitations } \\
\text { II }\end{array}$ \\
The activity is slightly limited. Asymptomatic at rest, daily activity classes cause obvious shortness of breath, \\
fatigue and palpitations
\end{tabular}




\section{Treatment method:}

A professional rehabilitation team took rounds of the selected patients daily and determined the intervening time of rehabilitation treatment according to the patient's condition within $24 \mathrm{~h}$. Rehabilitation training usually began within $2 \mathrm{~d}$ after the patient's vital signs were stable. Rehabilitation treatment team should first evaluate the patients who needed rehabilitation treatment by bedside for the first time. The team included senior professional rehabilitation physicians, rehabilitation therapists, ICU doctors and nurses to comprehensively assess patient's condition and master the occurrence,

TABLE 2: GENERAL INFORMATION OF REHABILITATION TREATMENT GROUP AND CONVENTIONAL TREATMENT GROUP

\begin{tabular}{lccc}
\hline Index & Rehabilitation group & Conventional treatment group & P value \\
\hline Age & $64.6 \pm 6$ & $65 \pm 6.6$ & 0.35 \\
gender & $21 / 22$ & $23 / 20$ & 0.66 \\
Body mass index & $23.7 \pm 2.1$ & $24 \pm 1.98$ & 0.42 \\
APACHEIl score (into the ICU) & $18 \pm 2.5$ & $18.58 \pm 2.5$ & 0.83 \\
Number of mechanical ventilation/total number of & $18(41.86)$ & $19(44.19)$ & 0.81 \\
groups (\%) & 11 & 10 & 0.82 \\
Acute exacerbation of COPD & 13 & 13 & 1.01 \\
Congestive heart failure & 6 & 7 & 0.76 \\
Sepsis & 7 & 8 & 0.77 \\
Acute cerebral hemorrhage & 6 & 5 & 0.73 \\
Acute cerebral infarction & & &
\end{tabular}

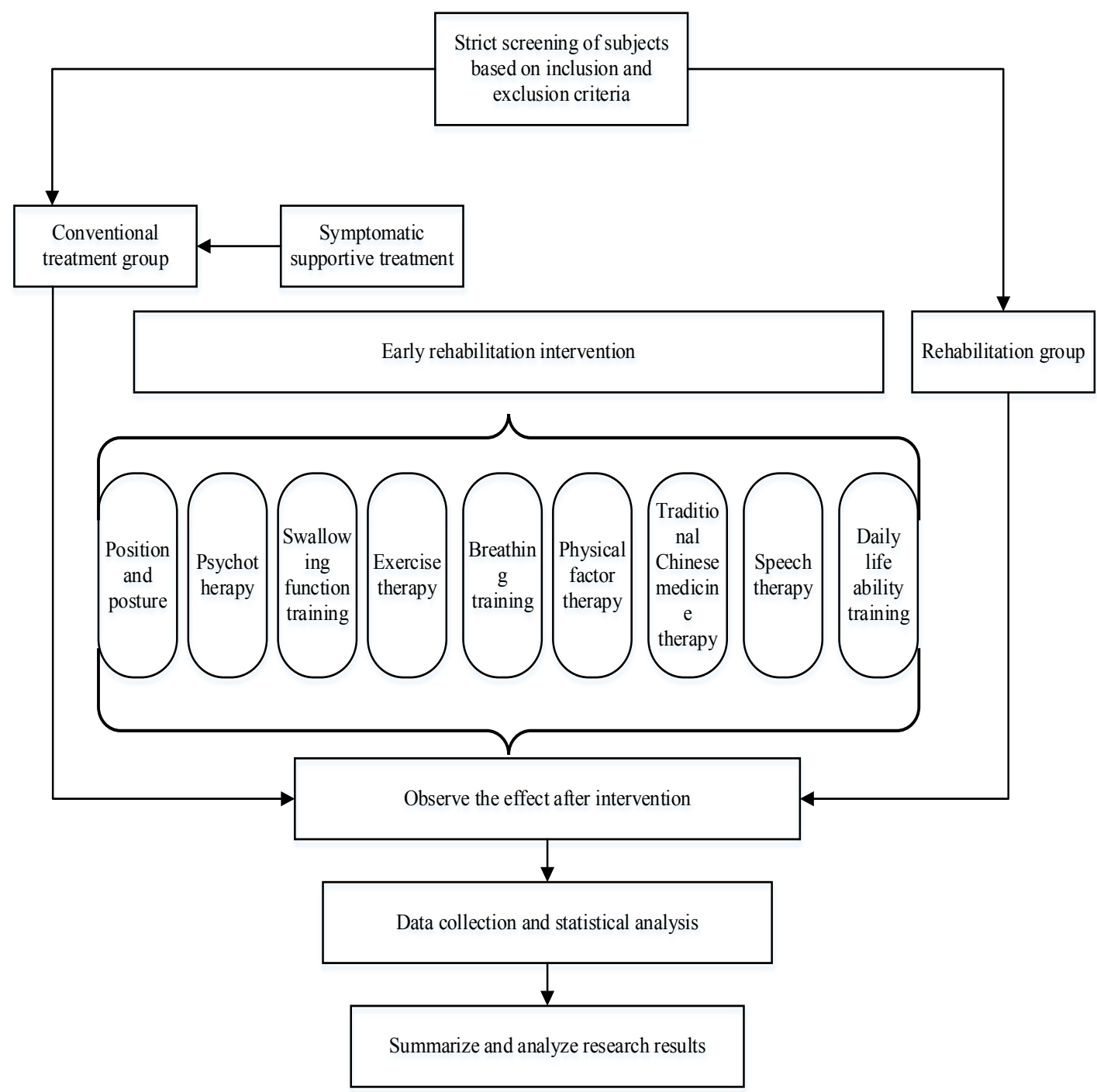

Fig. 2: Technical route of treatment 
development and prognosis of the disease. According to the patient's condition and basic diseases, all the patients who enter the rehabilitation group are comprehensively evaluated, and individualized rehabilitation training program is formulated. Comprehensive and progressive early rehabilitation training measures are adopted. The specific technical route is shown in fig. 2 .

According to the functional impairment possibly caused by the patient's illness, individualized comprehensive rehabilitation treatments such as exercise therapy, acupuncture, physiotherapy and psychotherapy were selected. The intensity of treatment is determined according to the patient's condition. The whole treatment process was based on the patient's difficulty to carry out targeted activities of daily exercises. According to the needs of the patients in the course of treatment, sedative drugs were used at night as far as possible. Before rehabilitation training, each patient was asked to stop taking sedative drugs for 1-2 $\mathrm{h}$. When Ramsay sedation score is $2 \mathrm{~min}$, the patients were given rehabilitation intervention treatment, as shown in Table 3. It was helpful for patients to complete mandatory actions with timely feedback of discomfort. Early rehabilitation treatment lasted from 10 to $30 \mathrm{~min}$ each time, twice a day or once a day and the rehabilitation process lasted more than $28 \mathrm{~d}$. If patients were discharged from the ICU, the specialized rehabilitation treatment of was still be provided by the same group of rehabilitation team.

\section{Rehabilitation treatment termination data:}

In the course of treatment, if any of the following conditions occurred, the training frequency, intensity, duration of training or suspension of the current rehabilitation program was implemented, and resting position was restored. If heart rate increased more than $30 \%$, when the patient was lying quietly, the heart rate

TABLE 3: RAMSAY SEDATION SCORE

\begin{tabular}{lc}
\hline Fraction & Performed \\
\hline 1 & Anxious \\
2 & Sober, quiet cooperation \\
3 & Drowsiness, quick response to instructions \\
4 & Light sleep state, can quickly wake up \\
5 & Fall asleep, slow response to the call \\
6 & Deep sleep, no response to the call \\
\hline
\end{tabular}

was lower than 40 beats/min or more than 110 beats/ min; if the blood pressure increased more than $20 \%$ in a quiet supine position, the average arterial pressure was less than $65 \mathrm{mmHg}$ or more than $110 \mathrm{mmHg}$; if the respiratory rate slowed down to less than 5 times/min or accelerated more than 40 times/min; $\mathrm{SpO}_{2}$ was less than $88 \%$ under oxygen inhalation; if the patient appeared dizzy, sweating, pale, extremely fatigued, angina, malignant arrhythmia or other discomfort symptoms or when the patient signaled to terminate the treatment.

In this study, SPSS15.0 statistical software was used to analyze the results. All measurements were expressed as mean \pm standard deviation (SD). Comparison is tested by T-test. Rates were compared by using the test and Fisher's exact probability method. In addition, Pearson correlation analysis is carried out for the related variables. Statistical analysis and evaluation are performed with $\alpha=0.05$.

\section{RESULTS AND DISCUSSION}

The incidence of adverse cardiovascular events such as time of treatment, tube prolapse, bed fall and deep venous thrombosis of lower extremities in the 2 groups were compared as shown in fig. 3. There was no significant difference in the incidence of adverse events such as arrhythmia, pressure sores and deep venous thrombosis between the rehabilitation group and conventional treatment group.

The APACHE II scores of patients in each group before and after treatment were compared as shown

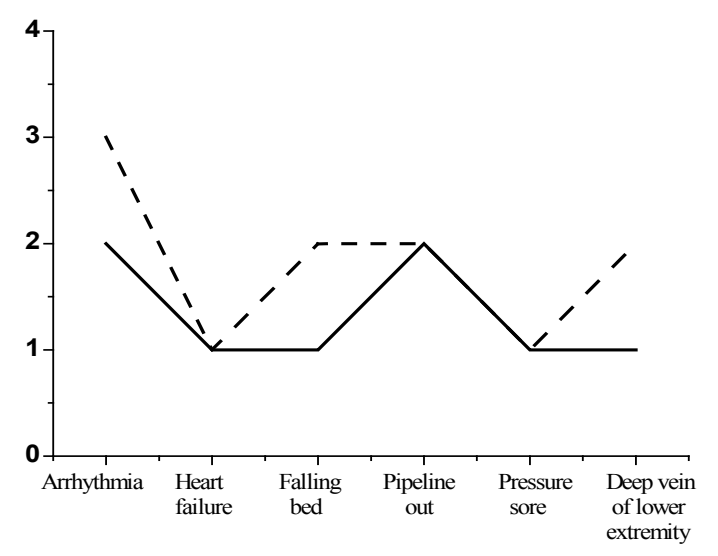

Fig. 3: Comparison of incidence of adverse events between the two groups

TABLE 4: COMPARISON OF APACHE II SCORES BEFORE AND AFTER TREATMENT AND BETWEEN THE TWO GROUPS

\begin{tabular}{lcccc}
\hline Project & Group & Before treatment & Day 28 after treatment & P value \\
\hline \multirow{2}{*}{ APACHEII score } & Rehabilitation group & $18.5 \pm 2.3$ & $11.4 \pm 1.65$ & 0 \\
& Conventional treatment group & $18.2 \pm 2.5$ & $11.01 \pm 1.74$ & 0 \\
& P value & 0.812 & 0.751 & 0 \\
\hline
\end{tabular}


TABLE 5: COMPARISON OF 28-DAY MORTALITY, ICU HOSPITALIZATION TIME, AND MECHANICAL VENTILATION TIME BETWEEN THE TWO GROUPS

\begin{tabular}{lccc}
\hline Project & Rehabilitation group & Rehabilitation group & P value \\
\hline 28-day mortality rate (\%) & $3(6.7)$ & $5(11.2)$ & 0.47 \\
ICU hospital stay (d) & $9.54 \pm 1.87$ & $10.2 \pm 2.13$ & 0.027 \\
Mechanical ventilation time (d) & $5.21 \pm 1.83$ & $6.23 \pm 1.434$ & 0.042 \\
\hline
\end{tabular}

in Table 3. After treatment, the APACHE II score in the rehabilitation group was significantly improved compared to that before treatment $(\mathrm{P}<0.05)$. After treatment, the APACHE II score in the conventional treatment group were also significantly improved $(\mathrm{P}<0.05)$.

The $28 \mathrm{~d}$ mortality rate, ICU hospitalization time and mechanical ventilation time were compared between the 2 groups as shown in Table 4 . The $28 \mathrm{~d}$ mortality in the rehabilitation group was lower than that in the conventional treatment group, but not significantly. The ICU hospitalization time and mechanical ventilation time in the rehabilitation group were significantly shorter than those in the conventional treatment group $(\mathrm{P}<0.05)$. The comparison of data before and after treatment in both groups, with in each group and among both groups is shown in Table 5. The BMI of both groups of patients before and after treatment was significant $(\mathrm{P}<0.05)$. The BMI of patients transferred to ICU in rehabilitation treatment group and conventional treatment group when compared were not significantly different. The BMI index of patients transferred from ICU in rehabilitation treatment group and the conventional treatment group was not significantly different from each other. The BMI of all patients before and after treatment was significantly different $(\mathrm{P}<0.05)$. After $28 \mathrm{~d}$ of individualized rehabilitation training, 40 critically ill patients in this group received early comprehensive rehabilitation treatment. Compared to the conventional treatment control group, they all achieved significant clinical outcome. The rehabilitation group has significantly improved limb activity and daily living skills. This study also showed that early rehabilitation treatment for critically ill patients does not increase patients heart rate. The incidence of adverse events such as arrhythmia, heart failure, deep venous thrombosis of lower extremities and the $28 \mathrm{~d}$ mortality of patients was also reduced as well as the duration of mechanical ventilation and ICU hospitalization, which clearly indicated that early comprehensive rehabilitation therapy was a safe and effective treatment regimen and cannot be replaced by drug therapy. Rehabilitation medicine in China started relatively late and progressed relatively slowly. So far, there are still many medical staff and patients who cannot correctly understand the importance and significance of rehabilitation treatment. With the limitation of rehabilitation conditions in domestic hospitals, a large number of patients miss the best opportunity of rehabilitation intervention. It is concluded that early rehabilitation exercises can significantly promote the functional recovery and prognosis of critically ill patients in ICU.

Early individualized and comprehensive rehabilitation intervention by professional rehabilitation team for critically ill patients in comprehensive ICU did not increase the 28-day mortality rate of critically ill patients or the incidence of adverse events. Early rehabilitation intervention was found to be safe. Compared to the conventional treatment, rehabilitation treatment shortened the ICU hospitalization time and mechanical ventilation time of critically ill patients. Both methods were however effective. Both could improve the basic living ability of patients, but early rehabilitation treatment improved the basic living ability of critically ill patients more effectively. It can be seen that early rehabilitation intervention for critically ill patients by professional rehabilitation team is safe and effective, which is conducive to the early return of patients to their families and society. Hospitals should strengthen the early rehabilitation treatment of critically ill patients, so as to further improve the quality of life of patients and reduce the disability rate.

\section{REFERENCES}

1. Cavalcanti AB, Bozza FA, Machado FR. Effect of a quality improvement intervention with daily round checklists, goal setting, and clinician prompting on mortality of critically ill patients: a randomized clinical trial. JAMA2016;315(14):148090.

2. Wischmeyer PE, McDonald D, Knight R. Role of the microbiome, probiotics, and 'dysbiosis therapy'incritical illness. Curr Opin Crit Care 2016;22(4):347.

3. Timsit JF, Azoulay E, Schwebel C. Empirical micafungin treatment and survival without invasive fungal infection in adults with ICU-acquired sepsis, Candida colonization, and multiple organ failure: the EMPIRICUS randomized clinical trial. JAMA 2016;316(15):1555-64.

4. Bloos F, Trips E, Nierhaus A. Effect of sodium selenite administration and procalcitonin-guided therapy on mortality in patients with severe sepsis or septic shock: a randomized clinical trial. JAMA Intern Med 2016;176(9):1266-76. 
5. Torres A, Sibila O, Ferrer M. Effect of corticosteroids on treatment failure among hospitalized patients with severe community-acquired pneumonia and high inflammatory response: a randomized clinical trial. JAMA 2015;313(7):67786.

6. Girardis M, Busani S, Damiani E. Effect of conservative vs conventional oxygen therapy on mortality among patients in an intensive care unit: the oxygen-ICU randomized clinical trial. JAMA 2016;316(15):1583-89.
This is an open access article distributed under the terms of the Creative Commons Attribution-NonCommercial-ShareAlike 3.0 License, which allows others to remix, tweak, and build upon the work non-commercially, as long as the author is credited and the new creations are licensed under the identical terms

This article was originally published in Special issue on "Trends in therapeutic Management of Various Conditions" Indian J Pharm Sci 2020:82(3) 